\title{
Editorial note to: \\ H. Weyl, \\ On the general relativity theory
}

\author{
Jürgen Ehlers
}

Published online: 5 June 2009

(C) Springer Science+Business Media, LLC 2009

Keywords General relativity $\cdot$ Cosmology $\cdot$ Golden Oldie

In Appendix III of the fifth edition of his book Space, Time, Matter [15] henceforth cited as W (which has not been translated into English), Hermann Weyl derived for the first time, motivated by cosmological considerations, the general frequency shift formula for sources and observers moving arbitrarily in a general spacetime. In his discussion of cosmology (W, 39) he argued for a non-stationary model. In support of this he cited astrophysical considerations (stellar ages and motions), used essentially Olbers' argument against Einstein's static model of 1917 [3], emphasized the significance of initial rather than (spatial) boundary conditions in GRT in relation to the difference between past and future, and asserted (in 1922!): "The present state of the system of stars on the sky does not resemble a final statistical state. The small stellar velocities are due to a common origin rather than to an adjustment, and it appears that the velocities between distant celestial objects on average increase with their mutual separations." (My translation and underlining.) Weyl then constructed a corresponding model, an isotropically expanding substratum which occupies an initially small, but monotonically growing part of de Sitter's hyperboloidal, globally non-stationary spacetime [7] depicted in Fig. 1, and applied to it his frequency shift formula. Using Slipher's red shift data and Lundmark's estimate of the distance of the Andromeda nebula, he obtained a 'world radius' (Hubble radius) of about 109 light years corresponding to a 'Hubble constant' of about $103 \mathrm{~km} / \mathrm{s} / \mathrm{Mpc}-6$ years ahead of

The republication of the original paper in translated version can be found in this issue following the editorial note and online via doi:10.1007/s10714-009-0826-6.

J. Ehlers (Deceased May 20, 2008)

Max-Planck-Institut für Gravitationsphysik (Albert Einstein Institute),

14476 Golm bei Potsdam, Germany

e-mail for correspondence: akr@camk.edu.pl 
Fig. 1

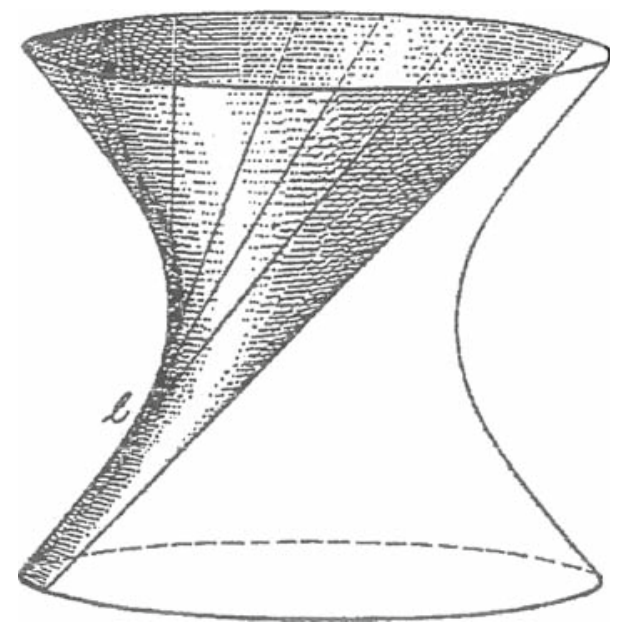

Hubble's path-breaking discovery which gave $530 \mathrm{~km} / \mathrm{s} / \mathrm{Mpc}$. (For the history of $H_{0}$ see, e.g., [14].)

Weyl's 'de Sitter model' is a kinematical one. Its material substratum has a welldefined motion and therefore exhibits a unique red shift, but its gravity is disregarded; the underlying spacetime metric satisfies Einstein's vacuum field equation with a cosmological constant. Before Weyl it was not clear which motion should be assigned to (test-) particles in de Sitter's spacetime which, by itself, does not represent a cosmological model; this confusion lasted at least until 1924 [2]. It is also worth noting that Friedmann published his first paper [6] presenting models containing expanding and gravitating matter, with or without a cosmological term, while Weyl completed the manuscript of W. The papers by Weyl and Friedmann contained the essential ingredients of expanding universe models, but they did not influence the development of cosmology, probably because of lack of observational data. The turn of the tides occurred around 1930 only (see, e.g. [5]).

Being aware of the possible astronomical importance of his results, Weyl published the note here reprinted, giving a proof of formula (2) which he had just stated in W.

In the remainder of this editorial I shall mention some additional historical facts and comment Weyl's text here reprinted. I follow the division into two sections used in that text.

(1) Until 1923 it was not clear how, according to GRT, proper frequencies or wavelengths are transferred from sources to observers, both possibly moving in a gravitational field. The rule used until then to compute frequency shifts applied to static spacetimes only, where a preferred global time coordinate exists, cf. [11,12,15]. The coordinate-independent formula (1) of the paper under review, derived by Weyl and independently at the same time by Lanczos [8], provided the general answer. It is based on von Laue's result [9] that in the geometrical optics approximation to Maxwell's equations the hypersurfaces of constant phase are null hypersurfaces and, consequently, light rays are null geodesics. Therefore, on world lines of sources and observers one has, in standard notation, $\omega=\mathrm{d} S / \mathrm{d} \tau=S,{ }_{\alpha} \mathrm{d} x^{\alpha} / \mathrm{d} \tau=k_{\alpha} U^{\alpha}$, which 
gives (1) as well as Schrödinger's equivalent formula [13]

$$
\frac{\omega_{\text {observed }}}{\omega_{\text {emitted }}}=\frac{(k \cdot U)_{\text {observed }}}{(k \cdot U)_{\mathrm{emitted}}}
$$

In the static or Robertson-Walker spacetimes (1) or $\left(1^{\prime}\right)$ give by now well known results.

(2) In W, Weyl had compared three conceptions about the world at large. 'Elementary cosmology' he called Minkowski spacetime occupied by a cosmic substratum whose world lines are parallel straight lines. 'Einstein cosmology' refers to Einstein's static, 'cylindrical' model of 1917. He named 'de Sitter cosmology' the one which I described above in the introductory part. There I also indicated the reasons why Weyl considered that model as the most satisfying among the three.

The geometrical construction of the collection of world lines of that model is given in the paper. To that I only add that such a set of world lines is characterized, according to $\mathrm{W}$, by the property that all of its members share the same causal future ('Wirkungsgebiet'). That, as far as I know, is the first use of causal structure in GRT. It implies that each particle can be influenced by all others at any time; in modern parlance there is no particle horizon. Moreover, the 'distance' of any particle A from another one, B, defined here as the proper length of the geodesic drawn from an event on $\mathrm{B}$, to one on $\mathrm{A}$, starting orthogonally to $\mathrm{B}$, tends to zero in the distant past. This together with the previous property is what Weyl expresses by saying that all particles of his model have a common origin and are causally connected - a very reasonable requirement revived only in connection with inflation [16]. (Side remarks: (i) Weyl's model, in which an 'exploding' substratum is surrounded by a vacuum, seems to me closer to the recent speculative idea of a universe arising out of a vacuum fluctuation, than a standard FLRW model. (ii) Bondi [1] connects with what he calls 'Weyl's postulate' the requirement of the existence of a universal time, which is not mentioned by Weyl and became clear only when Robertson-Walker coordinates were introduced, which was done for the matter part of Weyl's model by Lemaitre [10]. Bondi missed what was most important to Weyl, the 'causal coherence'.)

\section{Hermann Weyl: a brief biography}

By Andrzej Krasiński, abstracted from Ref. [17], with corrections provided by Hermann Weyl's son Michael and grandson Tom.

Hermann Weyl was born on 9 Nov 1885 in Elmshorn, near Hamburg, Germany. Already as a boy he showed a great talent for mathematics and for science. In 1904 he entered the University of Munich, where he took courses on mathematics and physics, and then went on to study the same topics at the University of Göttingen. He was completely captivated by Hilbert. He later wrote:

I resolved to study whatever this man had written. At the end of my first year I went home with the "Zahlbericht" under my arm, and during the summer vacation I worked my way through it-without any previous knowledge of 
elementary number theory or Galois theory. These were the happiest months of my life, whose shine, across years burdened with our common share of doubt and failure, still comforts my soul.

He got his doctorate from Göttingen, where his supervisor was Hilbert. He was awarded the degree in 1908, on the basis of the dissertation "Singuläre Integralgleichungen mit besonderer Berücksichtigung des Fourierschen Integraltheorems" ("Singular integral equations, with particular use of the Fourier integral theorems"). In Göttingen he held a teaching post as a Privatdozent until 1913. His habilitation thesis "Über gewöhnliche Differentialgleichungen mit Singularitäten und die zugehörigen Entwicklungen willkürlicher Funktionen" ("On ordinary differential equations with singularities, and the corresponding developments of arbitrary functions") investigated the spectral theory of singular Sturm - Liouville problems. He gave a lecture course on Riemann surfaces in the 1911-1912 session, and out of this course came his first book "Die Idee der Riemannschen Fläche" ("The idea of the Riemann surface"), which was published in 1913. It united analysis, geometry and topology, making rigorous the geometric function theory developed by Riemann. The book introduced for the first time the notions of a two-dimensional differentiable manifold, a covering surface, and the duality between differentials and 1-cycles. Weyl's idea of a space also included the separation property later introduced by and credited to Felix Hausdorff (1914). (This book was reprinted in 1997).

As a Privatdozent at Göttingen, Weyl had been influenced by Edmund Husserl who held the chair of philosophy there from 1901 to 1916.

Weyl had a talent for languages. He not only wrote beautifully in German-later he wrote English prose.

From 1913 to 1930 Weyl held the chair of mathematics at the Zürich Technische Hochschule. In his first academic year in this new post he was a colleague of Einstein who was at this time working out the details of the theory of general relativity. It was an event which had a large influence on Weyl who became fascinated by the mathematical principles lying behind the theory.

In 1915, being a German citizen, he was conscripted into the German army, but the Swiss government made a special request that he be allowed to return to his chair in Zürich, which was granted in 1916. In 1917 Weyl gave another course presenting an innovative approach to relativity through differential geometry. The lectures formed the basis of Weyl's second book "Raum-Zeit-Materie" which first appeared in 1918, with further editions in 1919, 1920 and 1923, each showing how his ideas were developing. These later ideas included a gauge metric (the Weyl metric), which led to a gauge field theory. (However Einstein, Pauli, Eddington, and others, did not fully accept Weyl's approach.) Over this period, Weyl also made contributions on the uniform distribution of numbers modulo 1, which are fundamental in analytic number theory.

In 1921 Schrödinger was appointed to Zürich, where he became the closest friend of Weyl. They shared many interests in mathematics, physics, and philosophy.

In 1923-1938 Weyl evolved the concept of continuous groups using matrix representations. In particular his theory of representations of semisimple groups, developed during 1924-1926, was very deep and considered by Weyl himself to be his greatest achievement. The ideas behind this theory had already been introduced by Hurwitz and 
Schur, but it was Weyl with his general character formula which took them forward. (Cartan also produced work of outstanding importance on this topic.)

Weyl was a guest lecturer at Princeton University 1928-1929. In 1931 he gave a series of three lectures (The Terry Lectures) at Yale University on the metaphysical implications of science, entitled "The Open World".

From 1930 to 1933 Weyl held the chair of mathematics at Göttingen, where he was appointed to fill the vacancy which arose on Hilbert's retirement. The rise of the Nazis persuaded him in 1933 to accept a position at the newly formed Institute for Advanced Study in Princeton, where Einstein also went. He remained there until his retirement.

Weyl undertook work of major importance at Princeton, but his most productive period were the years he spent at Zürich. He attempted to incorporate electromagnetism into the geometric formalism of general relativity. He produced the first unified field theory for which the Maxwell electromagnetic field and the gravitational field appear as geometrical properties of space-time. With his application of group theory to quantum mechanics he set up the modern subject. It was his lecture course on group theory and quantum mechanics in Zürich in session 1927-1928 which led to his third major text, "Gruppentheorie und Quantenmechanik", published in 1928.

We have seen above how Weyl's great works were first given as lecture courses. This was a deliberate design.

Several other great books by Weyl appeared during his years at Princeton. These include "Elementary Theory of Invariants" (1935), "The classical groups" (1939), "Algebraic Theory of Numbers" (1940), "Philosophy of Mathematics and Natural Science" (1949), "Symmetry" (1952), and "The Concept of a Riemannian Surface" (1955).

In 1951 Weyl retired from the Institute for Advanced Study. He described the Symmetry book as his 'swan song'. After his retirement Weyl and his wife Ellen spent part of their time at Princeton and part at Zürich. He died unexpectedly while in Zürich, on 8 Dec 1955, as he was walking home after posting letters of thanks to those who had wished him well on his seventieth birthday.

Weyl also worked on mathematical philosophy and the foundations of mathematics. He was much influenced by Husserl in his outlook, and also shared many ideas with Brouwer. Both shared the view that the intuitive continuum is not accurately represented by Cantor's set-theoretic continuum.

Weyl summed up his attitude to mathematics, writing:

My own mathematical works are always quite unsystematic, without mode or connection. Expression and shape are almost more important to me than knowledge itself. But I believe that, leaving aside my own peculiar nature, there is in mathematics itself, in contrast to the experimental disciplines, a character which is nearer to that of free creative art.

His often quoted comment:

"My work always tried to unite the truth with the beautiful, but when I had to choose one or the other, I usually chose the beautiful ..."

although half a joke, sums up his personality. 
Acknowledgment The Golden Oldie Editor is grateful to Hubert Goenner for locating Hermann Weyl's son Michael and grandson Tom, and getting their permission for this reprinting. Thanks are due also to Mr. Michael Weyl and Mr. Tom Weyl for reading the first draft of the biography and providing a few corrections.

\section{References}

1. Bondi, H.: Cosmology. Cambridge University Press, Cambridge (1950, 1960)

2. Eddington, A.S.: The Mathematical Theory of Relativity, 2nd edn. Cambridge University Press, Cambridge (1924)

3. Einstein, A.: Kosmologische Betrachtungen zur Allgemeinen Relativitätstheorie. Preuss. Akademie der Wissenschaften, Sitzungsber. 1917, p. 142

4. Einstein, A.: The Meaning of Relativity. Princeton University Press, Princeton (1921)

5. Ellis, G.F.R.: Innovation, resistance and change: the transition to the expanding universe. In: Bertotti, B., et al. (eds.) Modern Cosmology in Retrospect, pp. 97-113. Cambridge University Press, Cambridge (1990)

6. Friedman, A.: Über die Krümmung des Raumes. Z. Physik 10, 377 (1922) (reprinted, with historical comments: Gen. Relativ. Gravit. 31, 1985 (1999); +addendum: Gen. Relativ. Gravit. 32, 1937 (2000))

7. Klein, F.: Über die Integralform der Erhaltungssätze und die Theorie der räumlich geschlossenen Welt. Göttingen Nachrichten 394, 1918

8. Lanczos, C.: Über die Rotverschiebung in der de Sitterschen Welt. Z. Physik 17, 168 (1923)

9. von Laue, M.: Theoretisches über neuere optische Beobachtungen zur Relativitätstheorie. Physikalische Zeitschrift 21, 659 (1920)

10. Lemaitre, G.: Notes on de Sitter's universe. Phys. Rev. 25, 903 (1925)

11. von Laue, M.: Die Relativitätstheorie, Bd. 2: Die Allgemeine Relativitätstheorie und Einsteins Lehre von der Schwerkraft. Braunschweig (1921)

12. Pauli, W.: Relativitätstheorie. In: Encyklopedia der Mathematischen Wissenschaft, vol. 2 (1921)

13. Schrödinger, E.: Expanding Universes. Cambridge University Press, Cambridge (1950)

14. Tammann, G.A.: The ups and downs of the Hubble constant, pp. 1-29 in Rev. Mod. Astron. 19, Weinheim 2006

15. Weyl, H.: Raum, Zeit, Materie, 5th edn. Springer, Berlin (1923)

16. Abbott, L.F., Pi, S.-Y.: Inflationary Cosmology. World Scientific, Singapore (1986)

17. O’Connor, J.J., Robertson, E.F.: http://www-gap.dcs.st-and.ac.uk/ history/Biographies/Weyl.html (This text contains a large number of references to papers and books about Weyl and his results.) 